\title{
ARTIGOS
}

\section{ESTUDO RADIOLÓGICO LONGITUDINAL DO ESÔFAGO, EM ÁREA ENDÊMICA DE DOENÇA DE CHAGAS, EM UM PERÍODO DE SEIS ANOS}

\author{
Cleudson Castro, Vanize Macêdo, Jofre M. Rezende e Aluízio Prata
}

\begin{abstract}
Foi realizado estudo longitudinal de seis anos através do esofagograma, em 494 pessoas do projeto Mambai, das quais 212 (43\%) eram soropositivas. O estudo realizado em 1975/76 e 1980/82 constou em ambas as ocasiões, de duas abreugrafias do esôfago: a primeira, imediatamente após a ingestão de $75 \mathrm{ml}$ de solução baritada e outra 60 segundos após. Entre as 201 pessoas soropositivas normais no primeiro estudo, foram encontrados 4 (2\%) casos novos de megaesôfago do Grupo I, e entre as 11 com megaesôfago, 2 dos Grupos I e II evoluíràm, respectivamente, para os Grupos II e IV, indicando progressâo da esofagopatia em 2,8\% (6/212) dos soropositivos. Quatro pessoas com megaesôfago do Grupo I apresentaram esofagograma normalno segundo exame radiologico, com aparente "regressão" da esofagopatia. Chama a atençâo a existência de 10 pessoas com esofagogramas duvidosos no primeiro exame $e$, outras seis no segundo, sendo $75 \%$ delas soropositivas. Este achado pode estar indicando o esofagograma duvidoso como um marcador precoce da esofagopatia.
\end{abstract}

Palavras-chaves: Estudo longitudinaldomegaesôfago. Doençade Chagas. Abreugrafia do esôfago. Megaesôfago chagásico.

A doença de Chagas na forma cardíaca tern sido motivo de vários estudos longitudinais 15678 910111213 . Contudo, análises evolutivas sobre a esofagopatia e colopatia chagásicas são escassas, apesar de a doença de Chagas vir sendo estudada no campo, há décadas. O primeiro estudo longitudinal da esofagopatia chagásica foi feito por Macêdo ${ }^{7}$. Mais recentemente, outros têm sido publicados 561112 . O objetivo deste trabalho é apresentar o estudo evolutivo de seis anos, da esofagopatia chagásica, realizado no projeto Mambaí.

\section{MATERIAL E MÉTODOS}

Este trabalho é parte do projeto Mambaí, iniciado em 1975, que tem como objetivo o estudo clínico-epidemiológico da doença de Chagas. Em 1975/76, entre outros estudos, foram feitos

\footnotetext{
Núcleo de Medicina Tropical e Nutrição, Universidade de Brasilia, Brasília, DF e Faculdade de Medicina da Universidade Federal de Goiás, Goiânia, GO, Brasil.

Trabalho financiado pelo Conselho Nacional de Desenvolvimento. Científico e Tecnológico (CNPq).

Endereço para correspondência: Dr. Cleudson Castro. Núcleo de Medicina Tropical e Nutrição/UnB. CP: 04-671, 70919 970 Brasília, DF, Brasil.

Recebido para publicação em $27 / 05 / 92$.
}

esofagogramas em 2329 pessoas e, no período de $1980 / 82$, foram reexaminadas com o mesmo método e pelos mesmos investigadores, 558 indivíduos do projeto. Catalogando as pessoas que fizeram esofagogramas nas duas oportunidades e excluindo os exames ilegiveis, permaneceram em ambas as avaliações 494 pessoas, dos dois sexos, com idade, à época do estudo inicial, compreendida entre 4 e 79 anos, sendo a média de idade 24,7 anos. O estudo radiológico foi feito através de duas abreugrafias do esôfago com o paciente na posição oblíqua anterior direita, sendo a primeira radiografia realizada imediatamente depois da ingestão de 75 mililitros de sulfato de bário e a outra após 60 segundos. Foi utilizado filme de 70 milímetros nos exames de1975/ 76 e filmes de 35 e 70 milímetros, respectivamente, em 112 e 382 pessoas, nos exames de 1980/82.

As abreugrafias foram lidas conforme os critérios de Rezende e cols ${ }^{14}$ que estabeleceram quatro grupos com a seguinte difinição: Grupo I. Esôfagos de calibre aparentemente normal ao exame radiológico. Trânsito lento. Pequena retenção do contraste. Grupo II. Esôfagos com pequeno a moderado aumento de calibre. Apreciável retenção do contraste. Observamse com freqüência ondas terciárias associadas ou não à hipotonia do esôfago inferior. Grupo III. Esôfagos com grande aumento de calibre. Hipotonia do esôfago inferior. Atividade motora reduzida ou inaparente. 
Castro C, Macêdo V, Rezende JM, Prata A. Estudo radiológico longitudinal do esôfago, em área endêmica de doença de Chagas, em um periodo de seis.anos. Revista da Sociedade Brasileira de Medicina Tropical 25:225-230, out-dez, 1992.

Grande retenção de contraste. Grupo IV. Dolicomegaesôfagos. Esôfagos com grande capacidade de retenção, atônicos, alongados, dobrando-se sobre a cúpula diafragmática.

Houve um intervalo de, aproximadamente, sete anos entre a leitura dos exames realizados no período de 1975/76 e 1980/82. Em 1991, Rezende reviu ambas as radiografias de oito pessoas para dirimir dúvidas. O leitor das abreugrafias não teve conhecimento dos dados clínicos ou sorológicos dos pacientes.

À época, em que foi feita a primeira série de abreugrafias, foi realizada a sorologia para doença de Chagas, e em 1980/82 o exame foi repetido. Em ambas as oportunidades, foram feitas três reações sorológicas, em cada um dos dois laboratórios de referência.

\section{RESULTADOS}

Dentre os 494 indivíduos, $228(46,1 \%)$ tinham até 19 anos de idade. Em relação ao sexo, 255 $(51,6 \%)$ eram do sexo masculino e $239(48,4 \%)$ do sexo feminino. A sorologia feita em 1975/76, entre 492 pessoas mostrou que 212 (43\%) foram soropositivas, uma apresentou sorologia duvidosae 279 foram negativas, Tabela1. Na mesma Tabela, observa-se que os indivíduos com megaesôfago, exceto um, eram soropositivos. O estudo sorológico foi realizado em 1980/82 somente em 464 pessoas, das quais, $199(42,8 \%)$ foram positivas, duas foram duvidosas e 263 negativas, Tabela 2. Na mesma Tabela, observa-se que todas as pessoas com megaesôfago eram soropositivas.

$O$ intervalo entre os dois estudos radiológicos foi, no mínimo, de quatro anos para 21 pacientes e, no máximo, de sete anos para 201 indivíduos, sendo o intervalo médio de 6 anos para as 494 pessoas.

A distribuição dos resultados dos esofagogramas feitos em 1975/76, de acordo com a faixa etária, pode ser analisada na Tabela 3. Observa-se que até os 19 anos não houve esofagogramas alterados. A partir dos 20 anos, foram encontrados os esofagogramas duvidosos, aos 30 anos, os megaesôfagos do Grupo I e, após os 40 anos, um megaesôfago do Grupo II. Nos exames realizados em 1975/76 foram encontrados, 11 megaesôfagos do Grupo I e dois do Grupo II, com uma prevalência de $2,6 \%$ na população geral e de $6,1 \%$ entre os soropositivos. Dez pessoas apresentaram esofagogramas duvidosos.

Ainda na Tabela 3, pode ser observado entre os esofagogramas feitos em 1980/82 que, até a idade de 29 anos, houve apenas um esofagograma duvidoso. A partir dos 30, foram encontrados os megaesôfagos dos Grupos I e II e após os 40 anos, um megaesôfago do Grupo IV. De modo que nos exames realizados em $1980 / 82$ houve 10 megaesôfagos do Grupo I, dois do Grupo II e um do Grupo IV. Seis pessoas apresentaram esofagogramas duvidosos.

Das 17 pessoas que apresentaram megaesôfago, em 1975/76 e/ou 1980/82, 13 são do sexo masculino e quatro do sexo feminino.

Os megaesôfagos de sete pessoas não evoluíram durante o período de seguimento, sendo seis pessoas do Grupo I e uma do Grupo II. Houve três indivíduos com esofagograma normal e um com esofagograma duvidoso que passaram a ter megaesôfago do Grupo I, durante o periodo de estudo, Tabela 4 e Figura 1. Um indivíduo do Grupo I evoluiu para o Grupo II e um, do Grupo II progrediu para o Grupo IV. Também quatro indivíduos, cujas radiografias dos dois períodos foram revistas por Rezende,

Tabela 1 - Resultados dos esofagogramas de 1975/76 em relação à sorologia correspondente.

\begin{tabular}{|c|c|c|c|c|c|}
\hline \multirow{2}{*}{$\begin{array}{c}\text { Resultados } \\
\text { dos } \\
\text { Esofagogramas }\end{array}$} & \multicolumn{4}{|c|}{ Sorologia } & \multirow{2}{*}{ Total } \\
\hline & duvidosa & negativa & positiva & NR & \\
\hline Duvidoso & - & 2 & 8 & - & 10 \\
\hline Grupo I & - & 1 & 9 & 1 & 11 \\
\hline Grupo II & - & - & 2 & - & 2 \\
\hline Normal & 1 & 276 & 193 & 1 & 471 \\
\hline Total & 1 & 279 & 212 & 2 & 494 \\
\hline
\end{tabular}

$\mathrm{NR}=$ não realizada 
Castro C, Macêdo V, Rezende JM, Prata A. Estudo radiológico longitudinal do esôfago, em área endêmica de doença de Chagas, em um periodo de seis anos. Revista da Sociedade Brasileira de Medicina Tropical 25:225-230 out-dez, 1992.

Tabela 2 - Resultados dos esofagogramas de 1980/82 em relação à sorologia correspondente.

\begin{tabular}{lcccrr}
\hline $\begin{array}{l}\text { Resultados } \\
\text { dos }\end{array}$ & \multicolumn{3}{c}{ Sorologia } & \multirow{2}{*}{ Total } \\
\cline { 2 - 5 } Esofagogramas & duvidosa & negativa & positiva & NR & \\
\hline Duvidoso & - & 2 & 4 & - & 6 \\
Grupo I & - & - & 10 & - & 10 \\
Grupo II & - & - & 2 & - & 2 \\
Grupo IV & - & - & 1 & - & 1 \\
Normal & 2 & 261 & 182 & 30 & 475 \\
\hline Total & 2 & 263 & 199 & 30 & 494 \\
\hline
\end{tabular}

$\mathrm{NR}=$ não realizada

Tabela 3 - Resultados dos esofagogramas feitos em 1975/76 e 1980/82 de acordo com a faixa etária.

\begin{tabular}{|c|c|c|c|c|c|c|c|c|c|c|}
\hline \multirow{2}{*}{\multicolumn{2}{|c|}{$\begin{array}{l}\text { Faixa } \\
\text { etária } \\
(\text { anos }) *\end{array}$}} & \multicolumn{4}{|c|}{$1975 / 76$} & \multicolumn{5}{|c|}{$1980 / 82$} \\
\hline & & \multirow{2}{*}{$\frac{\text { Normal }}{115}$} & \multirow{2}{*}{$\frac{\text { Duvidoso }}{-}$} & \multirow{2}{*}{$\frac{\text { Grupo I }}{-}$} & \multirow{2}{*}{$\frac{\text { Grupo II }}{-}$} & \multirow{2}{*}{$\frac{\text { Normal }}{114}$} & \multirow{2}{*}{$\frac{\text { Duvidoso }}{1}$} & \multirow{2}{*}{$\begin{array}{c}\text { Grupo I } \\
-\end{array}$} & \multirow{2}{*}{ Grupo II } & \multirow{2}{*}{$\frac{\text { Grupo IV }}{-}$} \\
\hline 4 & -9 & & & & & & & & & \\
\hline 10 & -19 & 113 & - & - & - & 113 & - & - & - & - \\
\hline 20 & -29 & 83 & 3 & - & - & 86 & - & - & - & - \\
\hline 30 & -39 & 77 & 5 & 5 & - & 84 & - & 2 & 1 & - \\
\hline 40 & -49 & 40 & 2 & 2 & 2 & 39 & 3 & 2 & 1 & 1 \\
\hline 50 & -59 & 27 & - & 2 & - & 25 & 1 & 3 & - & - \\
\hline 60 & + & 16 & - & 2 & - & 14 & 1 & 3 & - & - \\
\hline Tot & & 471 & 10 & 11 & 2 & 475 & 6 & 10 & 2 & 1 \\
\hline
\end{tabular}

* Idade em 1975/76

Tabela 4 - Pacientes soropositivos que apresentaram evolução progressiva da esofagopatia no periodo de seis anos.

\begin{tabular}{|c|c|c|c|c|}
\hline \multirow{2}{*}{ Registro } & \multirow{2}{*}{ Idade* } & \multirow{2}{*}{ Sexo } & \multicolumn{2}{|c|}{$\begin{array}{l}\text { Resultados das } \\
\text { Abreugrafias }\end{array}$} \\
\hline & & & $1975 / 76$ & $1980 / 82$ \\
\hline 184 & 52 & F & normal & grupo I \\
\hline 190 & 46 & M & duvidoso & grupo I \\
\hline 1542 & 37 & $\mathrm{M}$ & grupo I & grupo II \\
\hline 1830 & 65 & $\mathbf{M}$ & normal & grupo I \\
\hline 3462 & 52 & $\mathrm{~F}$ & normal & grupo I \\
\hline 4044 & 47 & $\mathrm{M}$ & grupo II & grupo IV \\
\hline
\end{tabular}

* Idade em 1975/76

apresentaram aparente "Regressão" da esofagopatia, eram do Grupo I, e na radiografia de seguimento, foram classificados como normais (Figura 2), sendo que um desses, sempre apresentou sorologia negativa. Seis indivíduos que, no exame inicial eram normais, no exame de seguimento foram considerados como duvidosos e, nove pessoas que no exame de 1975/76 eram duvidosos, seis anos após foram classificados como normais, sendo $75 \%$ deles soropositivos.

\section{DISCUSSÃO}

O primeiro estudo longitudinal do megaesôfago foi feito por Macêdo ${ }^{7}$ avaliando 840 chagásicos durante cinco anos, dos quais 767 sem prévias alterações radiológicas do esôfago, 30 apresentaram esofagopatia indicando uma incidência de 3,9\%. Desses, 18 mostraram distúrbio da dinâmica esofágica e 12 megaesôfago. Coura e cols ${ }^{5} \mathrm{em}$ estudo longitudinal de 10 anos, em 110 chagásicos, observaram 3 casos novos, todos do Grupo I, o que dá uma incidência de $2,9 \%$ e dos casos de megaesôfago detectados no primeiro exame apenas um evoluiu no exame seguinte, do Grupo I para o Grupo II. Pereira e cols ${ }^{11}$ em estudo longitudinal de seis anos em 124 chagásicos, observaram cinco 
Castro C, Macêdo V, Rezende JM, Prata A. Estudo radiológico longitudinal do esôfago, em área endêmica de doença de Chagas, em um periodo de seis anos. Revista da Sociedade Brasileira de Medicina Tropical 25:225-230, out-dez, 1992.

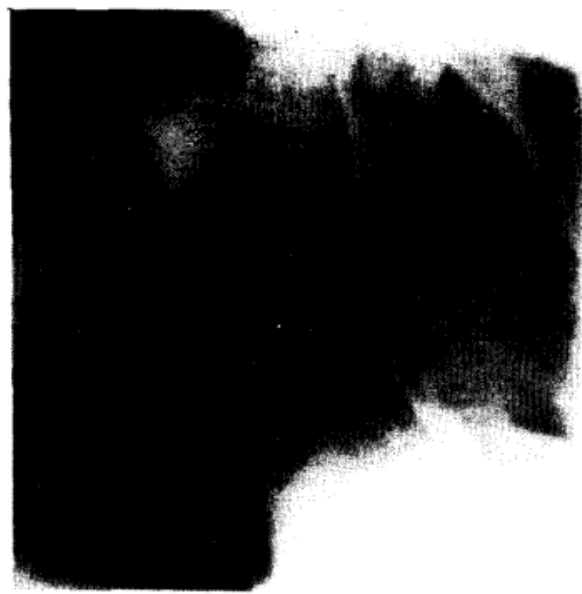

$1^{\text {a }}$

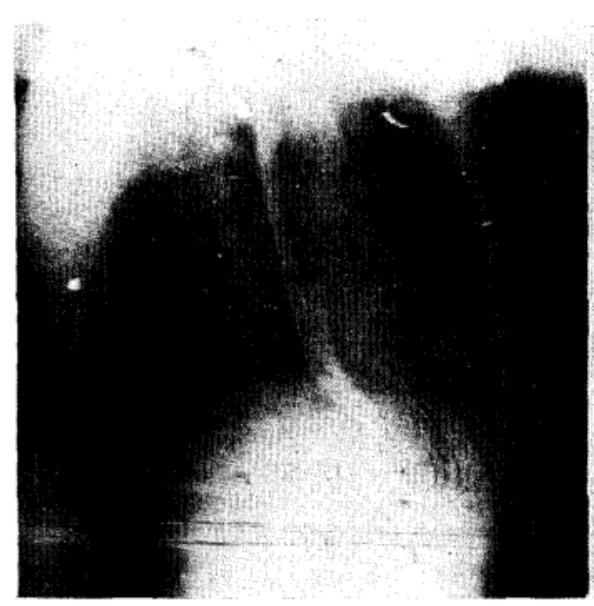

$1^{\mathrm{a}}$

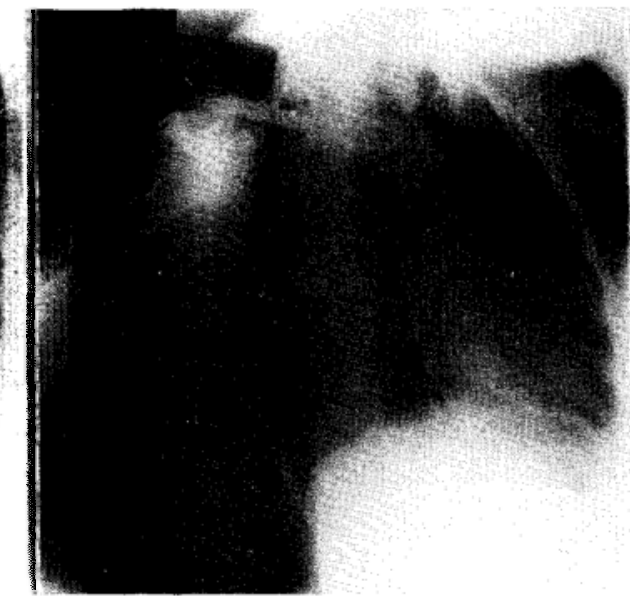

$2^{\mathrm{a}}$

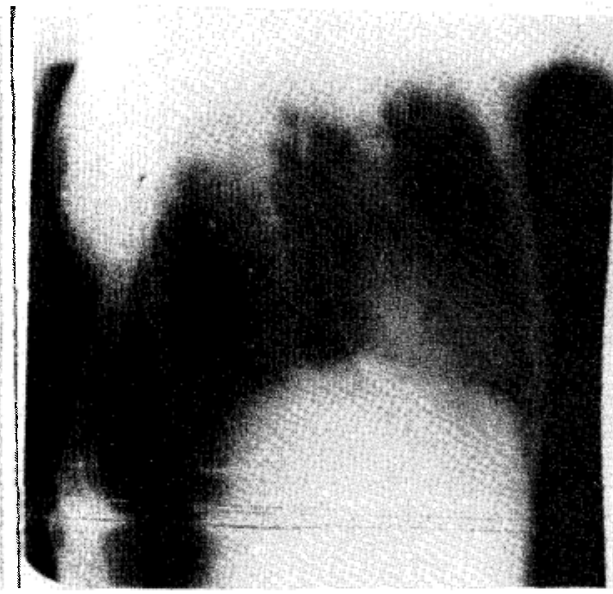

1981 $2^{a}$

Figura 1 - Abreugrafias do paciente VBS registro 184, feitas em 1975 (com aspecto normal) e em 1981 (mostrando evolução para megaêsofago do Grupo I).

$1^{a}$ Feita logo após a ingestão de $75 \mathrm{ml}$ de solução de sulfato de bário.

$2^{a}$ Feita um minuto após a primeira.

casos novos de megaesôfago todos do Grupo I, o que dá uma incidência de $4,5 \%$. Os megaesôfagos diagnosticados no primeiro exame permaneceram inalterados no exame de seguimento. Pompeu ${ }^{12}$, em estudo evolutivo de nove anos em 52 pessoas com infecção chagásica, encontrou três casos novos de megaesôfago, todos do Grupo I, o que dá uma incidência de 5,8\%. Dias, em estudo evolutivo de 115 pacientes a partir da fase aguda, entre 1940 e 1982, no município de Bambui ${ }^{6}$, encontrou 21 casos de esofagopatia, resultando em uma incidência de $18,3 \%$.
Neste estudo longitudinal de 6 anos realizado no projeto Mambaí, surgiram apenas quatro casos novos de megaesôfago, todos pertencentes ao Grupo I, sugerindo que a esofagopatia inicia gradual e lentamente. A incidência de esofagopatia nesse período entre os 201 indivíduos soropositivos que eram normais (incluindo os duvidosos) foi de $2 \%$ (4/201).

Por outro lado, quatro indivíduos que no primeiro exame foram classificados no Grupo I, no exame de revisão foram considerados com esofagograma normal. Pompeu ${ }^{12}$, fazendo estudo 
Castro C, Macêdo V, Rezende JM, Prata A. Estudo radiológico longitudinal do esôfago, em área endêmica de doença de Chagas, em um período de seis anos. Revista da Sociedade Brasileira de Medicina Tropical 25:225-230, out-dez, 1992.
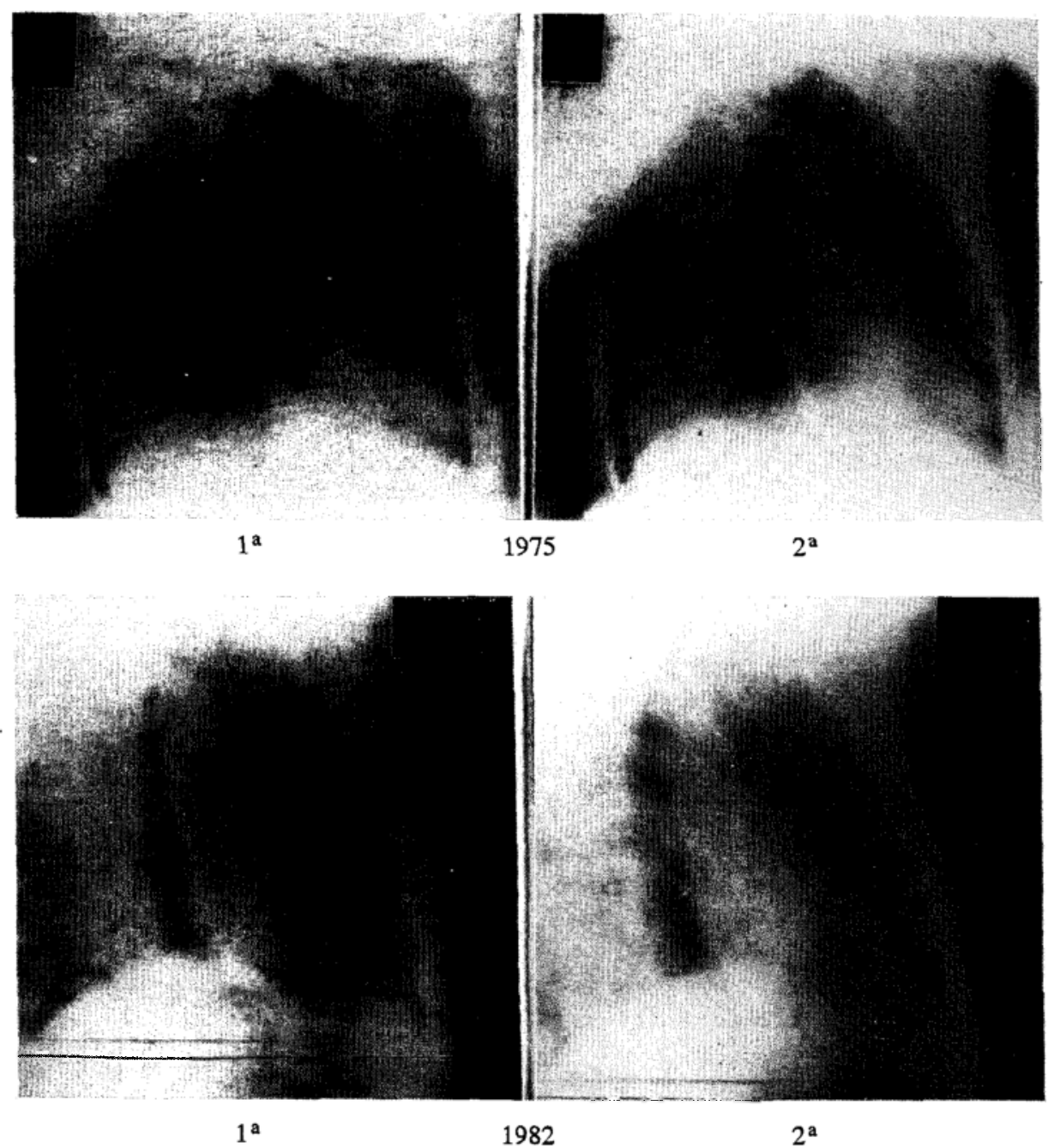

Figura 2 - Abreugrafias do paciente EFD registro 3203, feitas em 1975 (mostrando megaesôfago do Grupo I) e em 1982 (com aspecto normal).

$1^{a}$ Feita logo após a ingestão de $75 \mathrm{ml}$ de solução de sulfato de bário. $2^{a}$ Feita um minuto após a primeira.

evolutivo sobre a doença de Chagas no município de Luz, encontrou dois indivíduos que inicialmente tinham megaesôfago dos Grupos I e II, e nove anos apos apresentaram esofagograma normal. Comentando esses achados, o autor diz que "não houve regressão da esofagopatia preexistente e sim excesso de zelo do observador ao analisar os estudos radiológicos". Este fato ressalta que nem sempre é fácil distinguir um esofagograma normal do patológico, sendo necessário observar os critérios de normalidade estabelecidos por Cançado e cols ${ }^{2}$.

Há que considerar ainda o significado dos esofagogramas duvidosos mencionados de relance em trabalhos anteriores ${ }^{23}$. Como já foi assinalado, há indivíduos que têm esofagogramas alterados, mas não o suficiente para caracterizá-los como pertencentes ao Grupo I. São os esofagogramas duvidosos, sendo a maioria, de pessoas soropositivas ${ }^{4}$. Nesta situação houve 16 pacientes: 10 de 1975/76 e 6 de 1980/82, sendo $75 \%$ deles soropositivos. Temos dados sugerindo que estes indivíduos não devem ser considerados como normais, pois, provavelmente, com o prolongamento do estudo, eles evoluirão. 
Castro C, Macêdo V, Rezende JM, Prata A. Estudo radiológico longitudinal do esôfago, em área endêmica de doença de Chagas, em um periodo de seis anos. Revista da Sociedade Brasileira de Medicina Tropical 25:225-230, out-dez, 1992.

\section{SUMMARY}

A longitudinal study over six years was undertaken of 494 residents of the municipality of Mambai, Goiás. Two hundred and twelve (43\%) were seropositive in $1975 / 76$ and 199 of 464 of the same patients group, positive in 1980/82 (42.8\%). At both examination single radiographs of the oesophagus were obtained immediately after ingestion of $75 \mathrm{ml}$ of barium sulphate solution and a second X-ray taken one minute later. Among the 201 seropositive patients without megaesophagus in the first study 4 (2\%) evolved megaesophagus during the six years of observation. During this time, using Rezende's classification, patients with established megaesophagus, changed their group in the following manner. Orily one Group I patient changed to group II. Only one group II patient progressed to group IV. Progress of megaesophagus in the affected patients ocurred in $2.8 \%$ of 212 patients. Also four patients with grade $I$ megaesophagus initially had a normal oesophagogram on the follow up examination.

Ten patients had doubtful oesophagogram initially and six on follow up, $75 \%$ of these patients were seropositive. This study could indicate that a doubtful oesophagogram is an early sign of megaoesphagus.

Key-words: Longitudinal study of development of megaoesophagus. Chagas' disease. Barium swallow technique. Chagasic megaoesophagus.

\section{REFERÊNCIAS BIBLIOGRÁFICAS}

1. Alcantara A, Baruffa G, Aquino Neto JP, Olintho A, Savaldi T, Lassen C. Epidemiologia da doença de Chagas no RS. Revisão de pacientes após 10 anos de evolução. XIX Congresso da Sociedade Brasileira de Medicina Tropical p.35-37, 1983.

2. Cançado JR, Faria CAF, Ferreira SC. Abreugrafia contrastada para estudo epidemiológico da esofagopatia chagásica. Revista da Sociedade Brasileira de Medicina Tropical 11:167-176, 1977.

3. Castro C, Rezende JM, Camargo M, Prata A, Macêdo V. Prevalência de esofagopatia chagásica no município de Mambaí, Goiás - Brasil. Revista da Sociedade Brasileira de Medicina Tropical 20:1317, 1987.

4. Castro C, Rezende JM, Prata A, Macêdo V. Esofagogramas duvidosos em indivíduos com infecção chagásica. Esofagopatia inicial? ln: Pesquisa Aplicada em Doença de Chagas. Publicação da
Fundação Carlos Chagas, Araxá p.20, 1985.

5. Coura JR, Abreu LL, Pereira JB, Willcox HP. Morbidade da doença de Chagas. IV. Estudo longitudinal de dez anos em Pains elguatama, Minas Gérais, Brasil. Memórias do Instituto Oswaldo Cruz 80:73-80, 1985.

6. Dias JCP. Doença de Chagas em Bambuí, Minas Gerais, Brasil. Estudo Clínico epidemiológico a partir da fase aguda, entre 1940 e 1982 . Tese de Doutorado, Universidade Federal de Minas Gerais, Belo Horizonte, 1982.

7. Macedo VO. Influência da exposição à reinfecção na evolução da doença de Chagas. Revista de Patologia Tropical 5:33-116, 1976.

8. Maguire JH, Mott KE, Hoff R, Guimarães $A$, França JT, Souza JAA, Ramos WB, Sherlock IA. A three-year follow-up study of infection with Trypanosoma cruzi and eletrocardiographic abnormalities in a rural community in Northeast Brazil. American Journal of Tropical Medicine and Hygiene 31:42-47, 1982.

9. Moleiro F, Pifano F, Anselmi G, Ruesta A. La dinamica epidemiologica de la enfermedad de Chagas en el valle de los Naranjos. Estado Carabobo, Venezuela. Archivos Venezoclanos de Medicina Tropical y Parasitologia Medica 5:47-83, 1973.

10. Pereira JB, Cunha RV, Willcox HPF, Coura JR. Evolução da cardiopatia chagásica crônica humana no sertão do Estado da Paraíba, no período de 4, 5 anos. Revista da Sociedade Brasileira de Medicina Tropical 23:141-147, 1990.

11. Pereira JB, Willcox HP, Coura JR. Morbidade da doença de Chagas. III Estudo longitudinal de seis anos, em Virgem da Lapa-MG, Brasil. Memórias do Instituto Oswaldo Cruz 80:63-71, 1985.

12. Pompeu FR. Estudo longitudinal da doença de Chagas em trabalhadores rurais do município de Luz, Minas Gerais (1976-1985). Tese de Mestrado. Universidade Federal de Minas Gerais, Belo Horizonte, MG, 1990.

13. Puigbó JJ, Rhode JRN, Barrios HG, Yepez CG. Cuatro años de estudio longitudinal de una communidad rural con endemicidad chagasica. Boletin de la Oficina sanitaria Panamericana 66:112120, 1969.

14. Rezende JM, Lauar KM, Oliveira AR. Aspectos clínicos e radiológicos da aperistalse do esôfago. Revista Brasileira de Gastroenterologia 12:247$262,1960$. 